\title{
MEMS DEVICE INTEGRATED WITH VERTICALLY ALIGNED CARBON NANOTUBES FOR VIRUS CAPTURE AND DETECTION
}

\author{
Yin-Ting Yeh ${ }^{1}$, Nestor Perea-Lopez ${ }^{2}$, Yi Tang ${ }^{3}$, Brian U. McKellar ${ }^{1}$, Ramdane Harouaka ${ }^{1}$,Huaguang Lu ${ }^{3}$, \\ Mauricio Terrones ${ }^{2}$ and Si-Yang Zheng ${ }^{1} *$ \\ ${ }^{1}$ Department of Biomedical Engineering, ${ }^{2}$ Department of Physics, \\ ${ }^{3}$ Department of Veterinary and Biomedical Science, The Pennsylvania State University, U.S.A.
}

\begin{abstract}
Point-of-care virus diagnosis is highly desirable in worldwide infectious disease control. Here we report a hand-held device for capturing viruses by applying physical size based exclusion inside a point-of-care device integrated with vertically aligned carbon nanotube (VACNT) nanostructures to achieve label-free and high throughput virus capture. The microfluidic device is constructed from a VACNT channel wall synthesized bottom-up via chemical vapor deposition (CVD). The VACNT has $\sim 117 \mathrm{~nm}$ average gap size and $\sim 97 \%$ porosity. By bonding with a polydimethylsiloxane (PDMS) cover sealing the top, the aqueous sample containing virus particles filter through the VACNT channel wall under negative pressure applied at the outlet end. We have demonstrated that the device is capable of filtering $50 \mu \mathrm{L}$ of PBS containing $\sim 6.3 \times 10^{4}$ counts of lentivirus particles in 10 minutes with $97 \%$ of capture efficiency, quantified by the cell infectious titration technique.
\end{abstract}

\section{INTRODUCTION}

Once a viral infectious disease breaks out, the detriment can grow exponentially without effective quarantine. In 1918, an influenza outbreak caused 50 million deaths globally. The HIV/AIDS pandemic has resulted in 36 million deaths thus far and the number is increasing [1]. The emergence of these pathogens and their subsequent spread have not only had a destructive impact on global health but have also caused economic losses and interruptions in trade and travel [2]. Therefore, rapid on-site detection and analysis are crucial. Viruses are small parasites and display in a variety of sizes and shapes. In general, most discovered viruses range from 20-300 $\mathrm{nm}$ in size and contain either an RNA or DNA genome surrounded by a protective protein coat or capsid [3]. Current diagnostic approaches include direct virus detection via isolation of the virus in cell culture, identification of viral nucleic acids or antigens, and serological tests detecting virus-specific antibodies. However, viral culture requires a minimum of two to ten days of enrichment to provide statistically relevant information [4]. Because immunological approaches depend on specific recognitions provided by antibodies or probes [5], it poses a challenge to target unknown viruses or unidentified strains.

In this paper, we integrate a hand-held device to achieve virus capture and enrichment by size-based filtration. The active material is a VACNT forest with $97 \%$ porosity and the ability to selectively isolate virus from aqueous suspensions at the nanoscale. Via bottom-up synthesis processing, VACNT selectively grow on patterned iron catalyst thin films. First, we characterized VACNT material synthesis by scanning electron microscopy (SEM), transmission electron microscopy (TEM), and Raman spectroscopy. Second, we studied VACNT filtration properties of gap size and porosity and fitted the results using Darcy's equation. Third, we integrated droplet-shaped VACNT pattern into a microfluidic device via a sealing PDMS cover on top. We characterized the device filtration performance using lentivirus and measured the capture efficiency by cell infectious titration.

\section{FABRICATION AND FILTRATION MECHANISM \\ Bottom-up fabrication and device assembly}

Starting from a 4" prime silicon wafer, the silicon substrate was cleaned by piranha solution followed by subsequent sonication in acetone, IPA and DI water. In Fig.1A, The iron catalyst thin film was deposited by e-beam evaporation and patterned by photoresist lift-off process. The ion thin film is approximately $6.5 \mathrm{~nm}$ thick when measured by atomic force microscopy (AFM). Due to its better biocompatibility [6], nitrogen-doped CNTs were synthesized inside an aerosol based CVD by using benzylamine as the carbon source with nitrogen doping. The benzylamine mist was generated by an ultrasonic neubulizer and was carried by argon/hydrogen gas into two furnaces in series at $825^{\circ} \mathrm{C}$ under a flow rate of $2.5 \mathrm{~L} / \mathrm{min}$. In Fig.1B, the VACNT selectively grew on a patterned catalyst thin film to form an enclosed droplet shape microfluidic chamber. The newly synthesized VACNT pattern was then sealed on top with $\mathrm{s}$ molded PDMS chamber via oxygen plasma enhanced bonding. We fabricated the PDMS cover by using an SU-8 mold and by puncturing two fluidic access ports, one inlet and one outlet. In Fig. $1 \mathrm{C}$, the inlet port is attached with a $100 \mu \mathrm{L}$ cylindrical reservoir made with silicone. The outlet port is connected to a flow-through collection tube and a vacuum source through a T-shaped adaptor. The negative pressure is regulated by a mechanical regulator and measured with a pressure gauge.

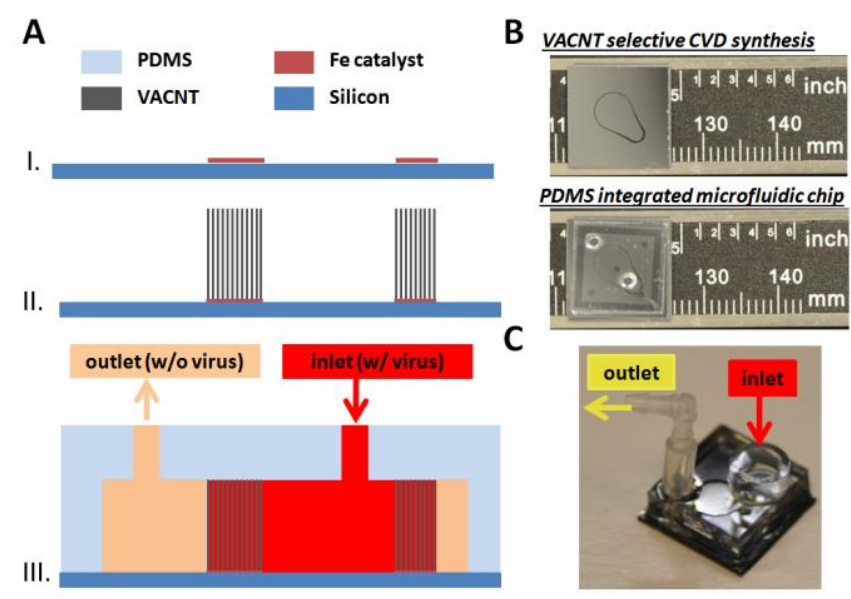

Figure 1. VACNT device fabrication and assembly process. A: Illustration of fabrication process flow. B: Top view of VACNT selective growth into droplet pattern (top), and device assembled with PDMS cover (bottom). C: Picture of hand-held virus capture device with fluidic access connections.

\section{Filtration mechanism and operation}

The test sample is loaded into the inlet reservoir by pipetting and actuated through the filtration device by negative pressure generated at the outlet end. The droplet-shaped porous wall was sealed on top by PDMS permanent bonding. Fig. 2 illustrates that the device performs dead-end filtration through the VACNT forest with nanoscale gap size and high porosity. During the filtration process, the suspension mixture containing particles of different sizes is 
transported through the VACNT microfluidic channel wall by the pressure driven flow. The particles with a size range similar to the gap size are trapped inside the membrane. Any larger particles are blocked and remain confined within the enclosed droplet chamber, while smaller particles flow through the VACNT membrane and are collected at the outlet.

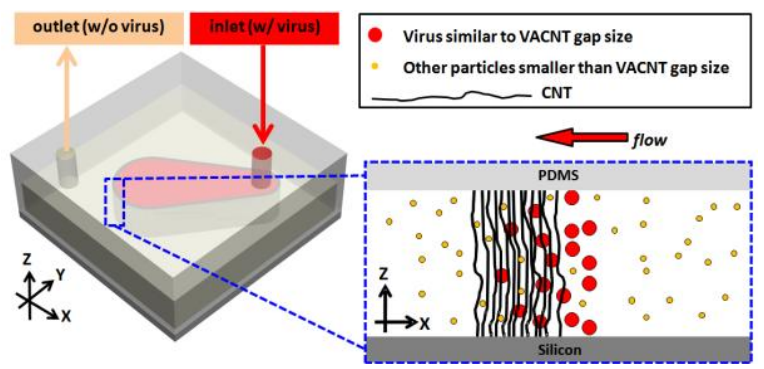

Figure 2. Schematic illustration of the virus isolation process inside the VACNT filtration device.

\section{RESULTS}

\section{VACNT material characterization}

We first characterized the newly synthesized VACNT by SEM, TEM and Raman spectroscopy. Fig.3A shows that multiwall carbon nanotubes (CNTs) selectively grow on the silicon substrate in a vertically aligned configuration with $\sim 50 \mu \mathrm{m}$ in height after a 30-minute CVD synthesis. Through image analysis with ImageJ, we plotted diameter measurements into a histogram distribution, as shown in Fig.3B. The results show that the diameter of VACNT ranges from 4.2 to $71.2 \mathrm{~nm}$ and is $25.3 \pm 11.8 \mathrm{~nm}$ on average.
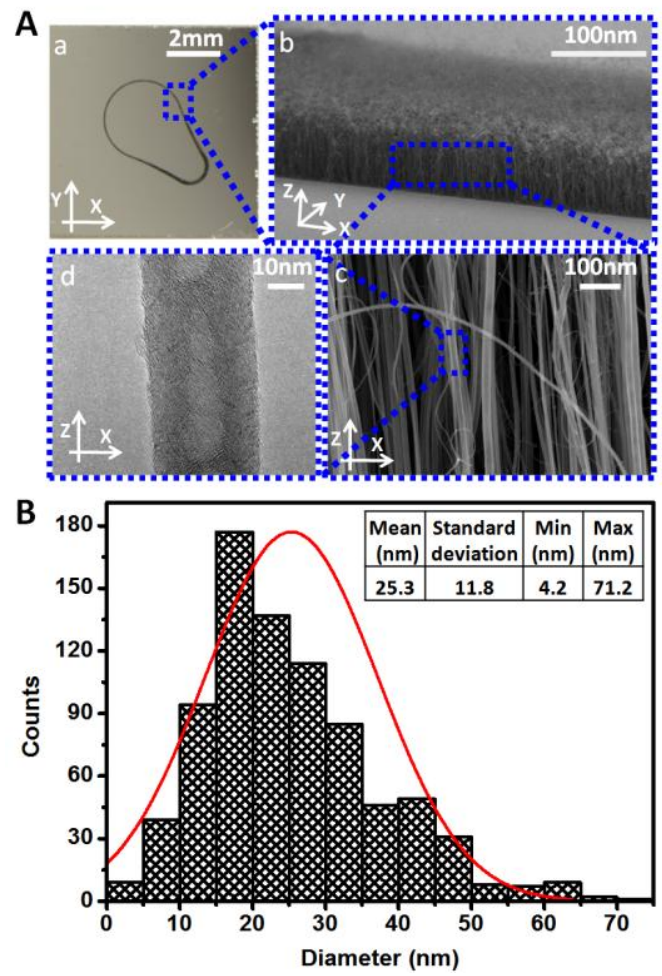

Figure 3. CVD synthesized VACNT material characterization. A: Images of VACNT synthesized in droplet shape pattern (a: bright field, b: SEM, c: SEM, and d: TEM images). B: Histogram of VACNT diameter and summary table of diameter distribution analysis.
Next, we used Raman microscopy (Renishaw, InVia Raman microscopy) to characterize the VACNT. We recorded the spectrum under $514 \mathrm{~nm}$ laser excitation for 30 seconds under 50X magnification. The laser power to the sample was $10 \mu \mathrm{W}$. As shown in Fig.4, the Raman spectrum indicates the VACNT has D, G and D* band peaks at 1352,1578 and $2659 \mathrm{~cm}^{-1}$, respectively. The results confirm that VACNT is multiple-wall with nitrogen doping [7].

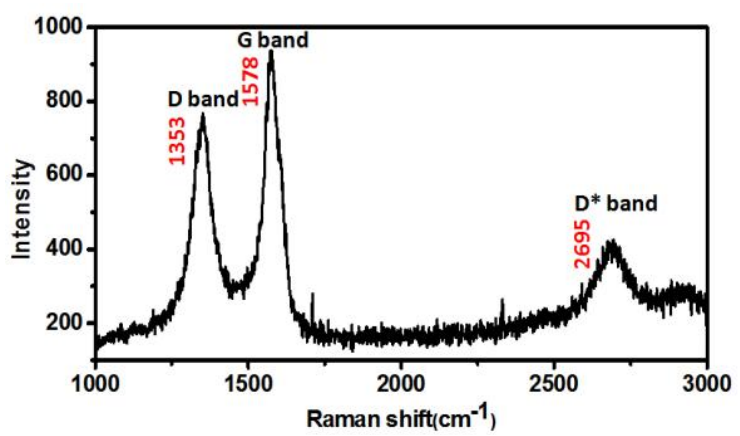

Figure 4, Raman spectrum of VACNT forest.

\section{Filtration properties}

Second, we studied the filtration properties (porosity and gap pore size) of VACNT. We synthesized VACNT on a $6.5 \mathrm{~nm}$ iron catalyst film with an increasing synthesis time of 5, 10, 20,30 and 40 minutes. Both diameter and density data were measured by image analysis of cross-sectional views of VACNT samples under SEM. For diameter analysis, the images were taken under $6 \times 10^{4}$ magnification and a total number of 200 focused CNTs were measured for each synthesis time. Fig.5A shows that the measurement results suggest the VACNT diameter can be considered independent of the synthesis time with $25.6 \pm 3.5 \mu \mathrm{m}$ on average. For density measurement, the images were taken under $2.5 \times 10^{4}$ magnification. By drawing a $1 \mu \mathrm{m}$ line perpendicular to VACNT growth direction on each image, we counted the numbers of focused CNTs that crossed with the drawing line. The results of the density measurement also indicate that density is independent of synthesis time after 10 minutes synthesis and the average density is $\sim 3.19 \times 10^{9}$ counts $/ \mathrm{cm}^{2}$. For the conditions of short synthesis time, such as 5 minutes, the random growth nature of the CNT synthesis at the early stage results in unpredictable VACNT density characterization [8].
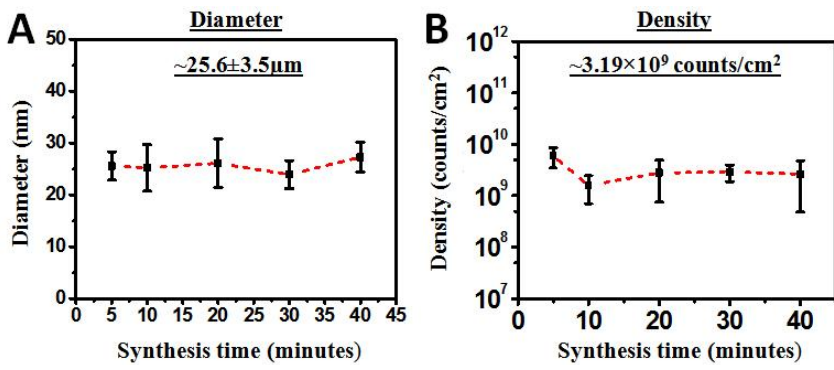

Figure 5. Results of VACNT characterization under different synthesis time by SEM image analysis $(n=6)$. A: Plot of diameter measurement results. B: Plot of density measurement results.

Next, we studied VACNT forest filtration properties by using Darcy's porous flow equation assuming the orientation of the VACNT forest as a cylindrical model with a uniform density and diameter [9]. In Fig.6, for the case of cylindrical pillars, the bulk porosity of the cylindrical array is described as below 


$$
\emptyset=1-\frac{\pi}{4} \times \frac{\mathrm{D}^{2}}{(\mathrm{P}+\mathrm{D})^{2}}
$$

where $\varnothing$ is the porosity, P is the gap pore size and D is the diameter of the cylindrical pillar [10]. As shown in Fig.6, the porosity is calculated by using density and diameter measurement obtained from Fig.4A and Fig.4B, respectively. The result shows the porosity of VACNT is approximately $97 \%$ on average.

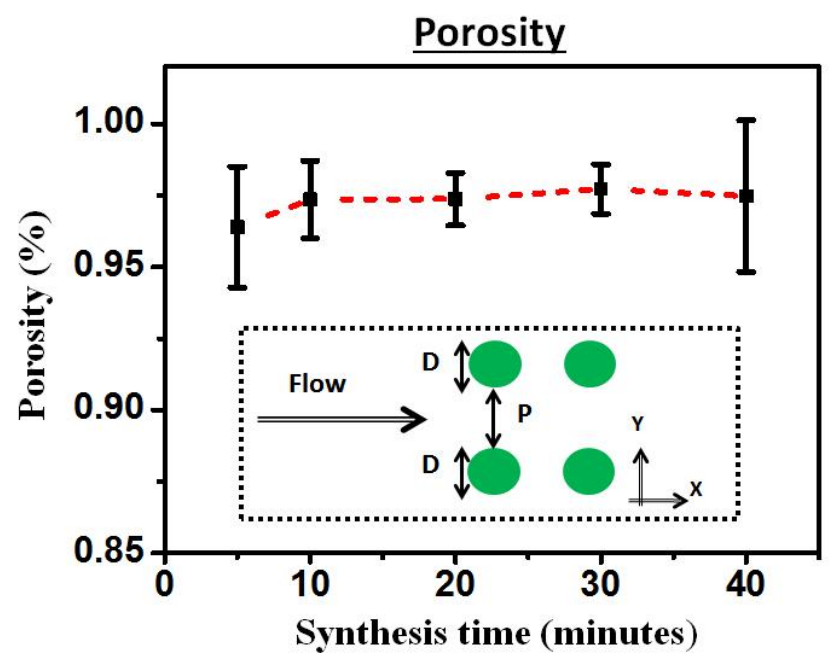

Figure 6. Results of VACNT porosity with illustration of cylindrical model assumption.

To calculated gap size $(\mathrm{P})$, we plugged porosity $(\phi)$, obtained based on cylindrical model assumption and the VACNT diameter (D) from Fig.5B into equation (1). As seen in Fig.7, the VACNT forest has gap size in the range of $92 \sim 136 \mathrm{~nm}$ with an average of $117.4 \pm 26.6 \mathrm{~nm}$.

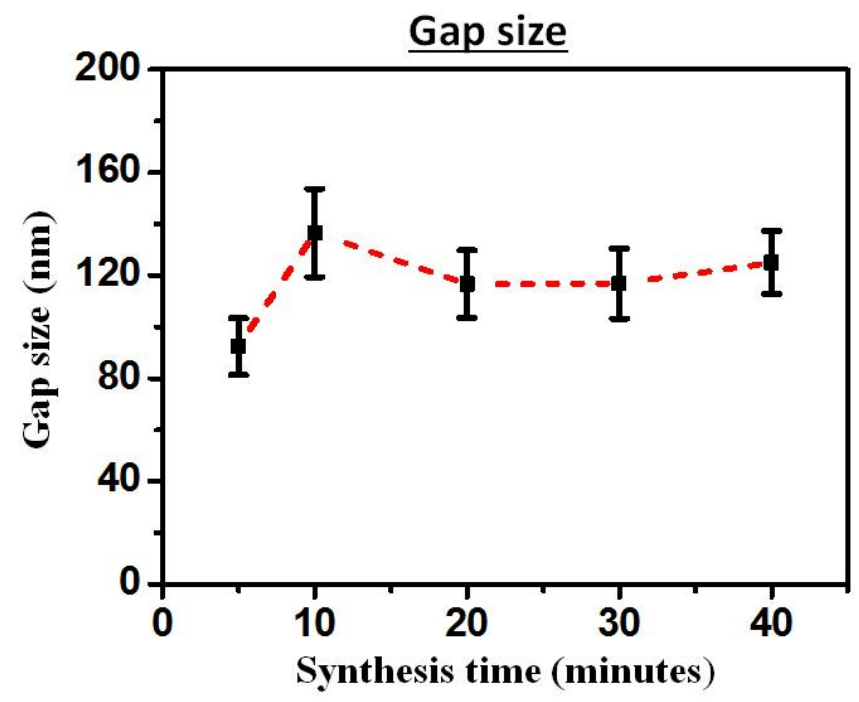

Figure 7. Calculated gap size of VACNT forest.

Feasibility study of virus capture

We tested the device feasibility of virus capture inside the VACNT integrated micro-device for lentivirus detection. We obtained lentivirus from HEK293 cell transfection [11]. The histogram plot of diameter distribution measured from SEM images shows that the lentivirus has dimensions of $128.5 \pm 24.1 \mathrm{~nm}$ in diameter. The inset of Fig.8 shows a SEM image with lentivirus particles fixed on a glass-slide after negative staining [12].

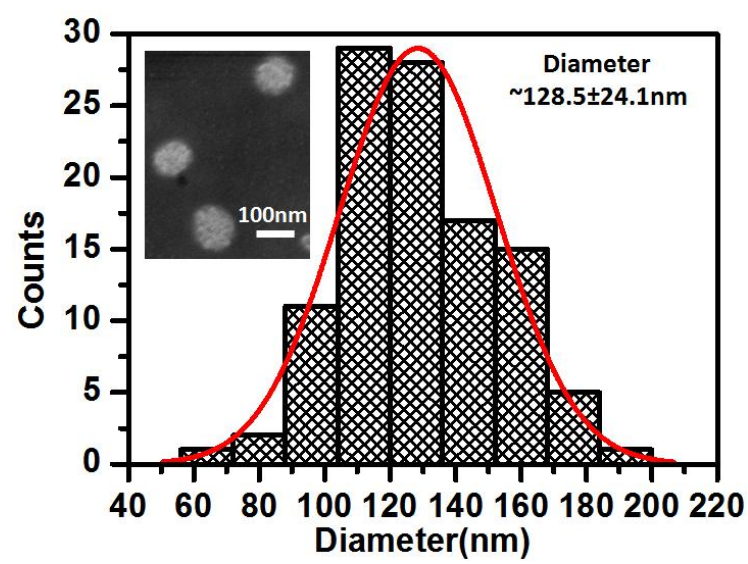

Figure 8, Histogram plot of lentivurs diameter measured via SEM images.

Prior to filtration testing, the virus sample was purified by ultracentrifugation, stained with the fluorophore Sybr Green (Invitrogen) and re-suspended in PBS with a final concentration of $\sim 10^{3} / \mu \mathrm{L}$. The VACNT filtration device is prepared by flushing with $0.5 \%$ Tween-20 $(50 \mu \mathrm{L})$ and PBS $(200 \mu \mathrm{L})$, respectively. During the filtration process, $50 \mu \mathrm{L}$ of fluorescently labeled virus was loaded into the inlet reservoir, and $200 \mu \mathrm{L}$ PBS was used to flush and wash away the non-trapped virus particles inside the device. In Fig.9 A and $\mathrm{B}$, a strong fluorescent signal is detected in the VACNT forest droplet shaped pattern, which indicates the presence of the virus. The trapped virus was confirmed by SEM, a seen in Fig.9 C and D.

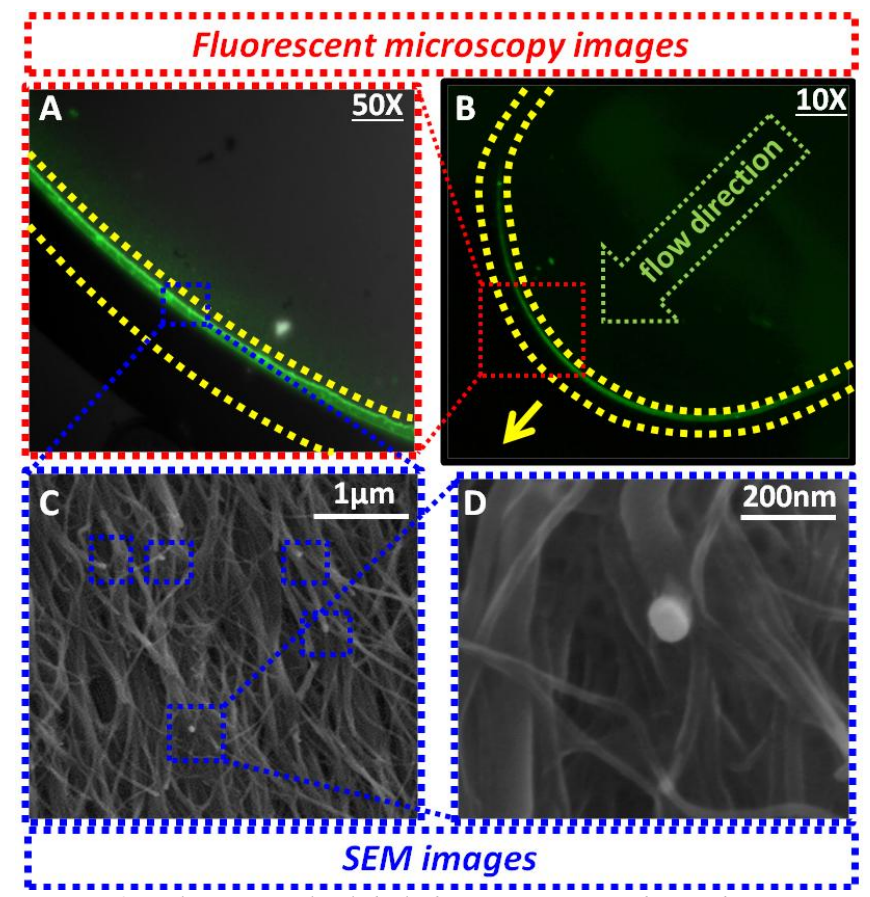
Figure 9. Fluorescently labeled virus captured inside VACNT filtration device. A,B: Fluorescent detection by fluorescence microscopy analysis (A: 50X magnification, B: 10X magnification). $C, D$ : SEM image of captured virus particle 


\section{Virus capture efficiency}

We further characterized the capture efficiency of the virus filtration device by counting the viral particles from sample flow-through collected at the outlet. We quantified the viral particle concentration by applying HEK293 cell infectious titration technique [13]. Cell culture plates containing HEK293 cell line with $100 \%$ confluence were infected by a series of dilutions of virus suspensions. As shown in Fig.10, the infected cells expressed green fluorescent protein (GFP). The fluorescent signal could be easily detected under fluorescence microscope. With the assumption of $100 \%$ infection efficiency of HEK293 cells by the lentivirus, we estimated virus particle concentration by multiplying the dilution factor to the counted number of infected cells. By loading a $50 \mu \mathrm{L}$ virus sample into the inlet of the device at a concentration of $6.3 \times 10^{4}$ counts $(100 \%)$ of total viral particles, we detected $1.8 \times 10^{3}$ counts $(3 \%)$ of viral particles inside flow-through to the outlet. Meanwhile, we tested two control experiments in parallel. The negative control experiment was to replace inlet virus sample with PBS and the positive control was to replace silicon substrate with VACNT forest into a prime silicon substrate without any pattern. We did not detect any virus for negative control experiment and $4.5 \times 10^{4}$ counts $(71 \%)$ of virus particles for the positive control.

A

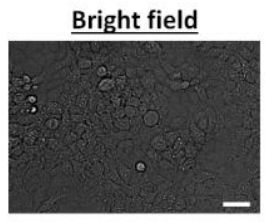

B

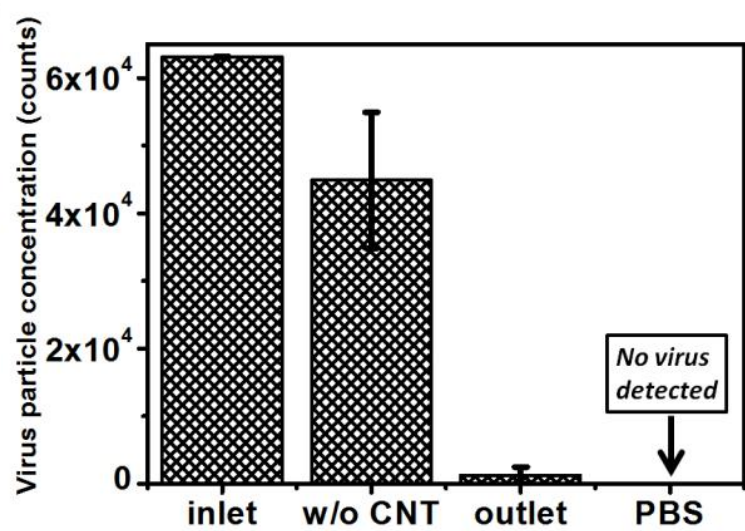

Figure 10, Capture efficiency characterization by HEK 293 cell infectious titration. A: Images of GFP protein expression detection via infected HEK 293 cell counting. B: Capture efficiency results characterized by virus concentration measurements.

\section{CONCLUSION}

We integrated a hand-held filtration device with $\sim 120 \mathrm{~nm}$ pore size, $\sim 97 \%$ porosity and $\sim 50 \mu \mathrm{m}$ high VACNT in a bottom-up synthesis process. We optimized the CVD synthesis process and characterized the filtration properties of the VACNT porous wall. We demonstrated that the lentivirus with $\sim 128 \mathrm{~nm}$ in diameter was captured inside the VACNT forest with capture efficiency of $97 \%$ by physical size exclusion. In point-of-care applications, the filtration process can be operated power-free if we replace the vacuum source with pipette suction. In addition, potential contaminations will be minimized since the trapped virus is contained inside the PDMS sealed device and is ready for further detection and manipulation. We believe that the device has potential in such point-of-care virus analysis applications.

\section{ACKNOWLEDGEMENT}

The authors appreciate assistance from the Penn State Material Characterization Lab (MCL). We would also like to thank Mr. Gene Gerber for his help with the experimental setup and also Dr. Yingwei Mao and Yijing Zhou for providing lentivirus particles. Travel support has been generously provided by the Transducer Research Foundation.

\section{REFERENCES}

[1] A. S. Fauci and D. M. Morens, "The perpetual challenge of infectious diseases," New England Journal of Medicine, vol. 366, pp. 454-461, (2012).

[2] M. S. Smolinski, M. A. Hamburg, and J. Lederberg, Microbial threats to health: emergence, detection, and response: National Academies Press, 2003.

[3] S. Baron and H. R. Gelderblom, "Structure and classification of viruses," in Medical Microbiology, ed Galveston, TX: University of Texas Medical Branch at Galveston, 1996.

[4] A. K. Shetty, E. Treynor, D. W. Hill, K. M. Gutierrez, A. Warford, and E. J. Baron, "Comparison of conventional viral cultures with direct fluorescent antibody stains for diagnosis of community-acquired respiratory virus infections in hospitalized children," The Pediatric infectious disease journal, vol. 22, pp. 789-794, (2003).

[5] I. M. Mackay, K. E. Arden, and A. Nitsche, "Real-time PCR in virology," Nucleic acids research, vol. 30, pp. 1292-1305, (2002).

[6] J. Carrero-Sanchez, A. Elias, R. Mancilla, G. Arrellin, H. Terrones, J. Laclette, et al., "Biocompatibility and toxicological studies of carbon nanotubes doped with nitrogen," Nano Letters, vol. 6, pp. 1609-1616, (2006).

[7] S. Webster, J. Maultzsch, C. Thomsen, J. Liu, R. Czerw, M. Terrones, et al., "Raman characterization of nitrogen doped multiwalled carbon nanotubes," in MATERIALS RESEARCH SOCIETY SYMPOSIUM PROCEEDINGS, 2003, pp. 129-134.

[8] M. Bedewy, E. R. Meshot, H. Guo, E. A. Verploegen, W. Lu, and A. J. Hart, "Collective Mechanism for the Evolution and Self-Termination of Vertically Aligned Carbon Nanotube Growth," The Journal of Physical Chemistry C, vol. 113, pp. 20576-20582, 2009/12/03 (2009).

[9] F. Fachin, G. D. Chen, M. Toner, and B. L. Wardle, "Integration of Bulk Nanoporous Elements in Microfluidic Devices With Application to Biomedical Diagnostics," Microelectromechanical Systems, Journal of, vol. 20, pp. 1428-1438, (2011).

[10]K. Vafai, Handbook of porous media: Crc Press, 2010.

[11]X. Wang and M. McManus, "Lentivirus Production," p. e1499, 2009/10/02/ (2009).

[12]M. Laue, "Electron microscopy of viruses," Methods in cell biology, vol. 96, pp. 1-20, (2010).

[13]L. Sastry, T. Johnson, M. Hobson, B. Smucker, and K. Cornetta, "Titering lentiviral vectors: comparison of DNA, RNA and marker expression methods," Gene therapy, vol. 9, (2002).

\section{CONTACT}

*S.Y. Zheng, tel: +1-814-865-8090; sxz10@psu.edu 